\title{
BMJ Open Vascular flow density in pathological myopia: an optical coherence tomography angiography study
}

\author{
Jing Mo, ${ }^{1}$ Anli Duan, ${ }^{1}$ Szyyann Chan, ${ }^{1}$ Xuefei Wang, ${ }^{1}$ Wenbin Wei ${ }^{1,2}$
}

To cite: Mo J, Duan A, Chan S, et al. Vascular flow density in pathological myopia: an optical coherence tomography angiography study. BMJ Open 2017;7: e013571. doi:10.1136/ bmjopen-2016-013571

- Prepublication history for this paper is available online To view these files please visit the journal online (http://dx.doi.org/10.1136/ bmjopen-2016-013571)

Received 24 July 2016 Revised 4 November 2016 Accepted 12 December 2016

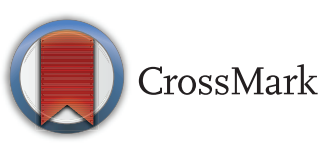

${ }^{1}$ Beijing Ophthalmology and Visual Science Key Lab, Beijing Tongren Eye Center, Beijing Tongren Hospital, Capital Medical University, Beijing, China

${ }^{2}$ Beijing Key Laboratory of Intraocular Tumor Diagnosis and Treatment, Beijing, China

Correspondence to Professor Wenbin Wei; weiwenbintr@163.com

\section{ABSTRACT}

Objectives: To investigate vascular flow density in pathological myopia with optical coherence tomography (OCT) angiography.

Design: A prospective comparative study was conducted from December 2015 to March 2016.

Setting: Participants were recruited in Beijing Tongren Hospital.

Participants: A total of 131 eyes were enrolled, which were divided into three groups: 45 eyes with emmetropia (EM; mean spherical equivalent (MSE) $0.50 \mathrm{D}$ to $-0.50 \mathrm{D}), 41$ eyes with high myopia (HM; MSE $\leq-6.00 \mathrm{D}$, without pathological changes), and 45 eyes with pathological myopia (PM; MSE $\leq-6.00 \mathrm{D}$ and axial length $(A L) \geq 26.5 \mathrm{~mm}$, and with pathological changes).

Main outcome measures: Macular, choriocapillaris and radial peripapillary capillary (RPC) flow densities were measured and compared between groups, and their relationships with $\mathrm{AL}$ and best corrected visual acuity (BCVA) were analysed.

Results: Significant differences were found in macular, choriocapillaris and RPC flow densities among the three groups $(p<0.05)$. Multiple comparisons revealed that, compared with the EM and HM groups, macular and RPC flow densities of the PM group were significantly decreased $(p<0.05)$, but no significant difference in choriocapillaris flow density was found between the PM and HM groups $(p=0.731)$. Compared with the EM group, retinal flow density in the macular and arcuate fibre region was not decreased in the HM group. In addition, there was a negative correlation between $A L$ and superficial macular flow density $(\beta=-0.542, p<0.001)$, deep macular flow density $(\beta=-0.282, p=0.002)$ and RPC flow density $(\beta=$ $-0.522, p<0.001$ ); and a positive correlation between BCVA and superficial macular flow density $(\beta=0.194$, $p=0.021)$, deep macular flow density $(\beta=0.373$, $p<0.001)$, and choriocapillaris flow density $(\beta=0.291$, $\mathrm{p}=0.001$ )

Conclusions: Macular and RPC flow densities decreased in pathological myopia compared with high myopia and emmetropia. No significant decrease of retinal flow density in the macular and arcuate fibre region was found in high myopic eyes compared with emmetropic eyes. Moreover, macular and RPC flow densities were negatively related to $\mathrm{AL}$, and macular flow density was positively related to BCVA.

\section{Strengths and limitations of this study}

- This study investigated the vascular flow density in pathologically myopic eyes with optical coherence tomography angiography.

- This study was limited by its cross-sectional design, single-centre and monoracial background.

- The mechanisms of the decreased ocular perfusion and how to preserve or improve perfusion in pathological myopic eyes required further study.

\section{INTRODUCTION}

Pathological myopia (PM) is one of the major causes of visual impairment and legal blindness worldwide, ${ }^{1}{ }^{2}$ especially in East Asia. $^{3-5}$ The principal alterations in $\mathrm{PM}$ include excessive axial elongation of the globe and associated deformation of the posterior ocular segment, with posterior staphyloma as an important primary sign. Secondary to the increased axial length (AL) and staphyloma formation, a range of retinal and choroidal lesions may develop in the posterior pole in eyes with PM. $^{6}{ }^{7}$ Several studies have revealed vascular changes in high myopia (HM) and PM, and such changes may be related to the pathogenesis of PM. ${ }^{8-11}$

Different techniques have been used to study retinal or choroidal blood flow in myopic eyes. Fluorescein angiography (FA) has been used to observe ocular circulation in myopia, but FA could not image the radial peripapillary or deep capillary networks well. $^{8} 12$ Indocyanine green angiography (ICGA) was used to observe retrobulbar vessels and choroidal vasculatures in PM, but ICGA could not visualise the microvasculature well. ${ }^{13}$ In addition, FA and ICGA were not commonly used in myopia because of their invasive nature and difficulty in quantification. Colour Doppler imaging (CDI) has been used to obtain precise measurements of choroidal and retinal blood flow, ${ }^{9}$ but it was 
not sensitive enough to accurately measure the macular and peripapillary microcirculation. Optical coherence tomography (OCT) angiography with split-spectrum amplitude-decorrelation angiography (SSADA) is a recently developed clinical tool, which has allowed for non-invasive and quantitative investigation of retinal and choroidal vasculature, including the microvasculature. $^{12}$ 14-16

The purpose of this study was to investigate the macular and peripapillary vascular flow density of pathologically myopic eyes with OCT angiography, and to determine whether ocular circulation was altered in the pathologically myopic eye.

\section{METHODS}

Participants

This observational, cross-sectional study included 45 eyes with PM and 41 eyes with HM, which were enrolled from December 2015 to March 2016 in the department of ophthalmology in Beijing Tongren Hospital. At the same time age-matched and sexmatched 45 healthy control eyes were recruited. Written informed consents were obtained from all study participants.

The study eyes were divided into three groups: (1) emmetropia (EM; mean spherical equivalent (MSE) $0.50 \mathrm{D}$ to $-0.50 \mathrm{D}$ ); (2) HM (MSE $\leq-6.00 \mathrm{D}$, and without pathological changes); (3) PM (MSE $\leq-6.00 \mathrm{D}$ and AL $\geq 26.5 \mathrm{~mm}$, and according to the definition and classification (five categories) stated by the 'META analysis for Pathologic Myopia (META-PM)' study group classification $^{6} \mathrm{PM}$ was defined as eyes having chorioretinal atrophy equal to or more severe than diffuse atrophy).

Exclusion criteria included: (1) having other ocular diseases such as glaucoma or any retinal vascular disease; (2) a history of systemic diseases such as diabetes mellitus which might affect the ocular circulation; (3) a history of intraocular surgery, laser photocoagulation or ocular injury.

\section{Examination}

All study participants underwent a complete ophthalmic examination, which included measurement of best corrected visual acuity (BCVA), refractive status assessment using an automatic refractometer (Auto Refractometer RM-8900 Topcon, Tokyo, Japan), calculation of the MSE using the spherical dioptre plus one-half of the cylindrical dioptre, intraocular pressure (IOP) measurement using a non-contact tonometer (Full Auto Tonometer TX-F; Topcon, Tokyo, Japan), slit-lamp assisted biomicroscopy (Haag-Streit, 3098 Koeniz, Switzerland) and fundus photography (Hybrid Digital Mydriatic Retinal Camera CX-1 Canon, Tokyo, Japan), and AL measurement using IOL Master (CarlZeiss, Jena, Germany). Blood pressure was also measured.

OCT angiography scans were obtained using RTVue XR OCT (Optovue, Fremont, California, USA, Software
V.2015.100.0.35.) with the angio retina mode $(3 \times 3 \mathrm{~mm})$ and the angio disc mode $(4.5 \times 4.5 \mathrm{~mm})$. The technique of OCT angiography including the SSADA method has been described in detail recently. ${ }^{14-16}$ Vascular flow density was defined as the percentage area occupied by vessels.

Exclusion criteria for OCT angiography scans included: (1) signal strength index <40; (2) low quality of images with severe artefacts because of poor fixation; (3) PM with epiretinal membrane, foveoschisis, macular holes, choroidal neovascularisation and retinal detachment; these complications might interfere with the automatic layer segmentation software.

\section{Retinal density of macular angiogram}

For measurement of retinal density, a $3 \times 3 \mathrm{~mm}$ macular angiogram of superficial and deep layers was analysed using Optovue software with density function. Each of the layers was automatically segmented by the software: superficial retinal layer extending from the inner limiting membrane with an interface offset of $3 \mu \mathrm{m}$ to the inner plexiform layer (IPL) with an interface offset of $16 \mu \mathrm{m}$; deep retinal layer extending from the IPL with an interface offset of $16 \mu \mathrm{m}$ to the IPL with an interface offset of $69 \mu \mathrm{m}$. Flow density was separately calculated in five regions (fovea, tempo, superior, nasal and inferior) based on the ETDRS (Early Treatment Diabetic Retinopathy Study) contour (figure 1A). The whole en face flow density and parafovea flow density were also measured.

\section{Choriocapillary density of macular angiogram}

For measurement of choriocapillary density, a $3 \times 3 \mathrm{~mm}$ macular angiogram of the choriocapillary layer (from RPE with an RPE offset of $31 \mu \mathrm{m}$ to the deeper layer with an RPE offset of $59 \mu \mathrm{m}$ ) was analysed using Optovue software with flow function. Choriocapillary density was calculated as vessel areas of choriocapillaris divided by selected areas $\left(9 \mathrm{~mm}^{2}\right)$.

\section{Radial peripapillary capillary flow density of disc angiogram}

For measurement of parameters of the disc angiogram, a $4.5 \times 4.5 \mathrm{~mm}$ large en face image was analysed with Optovue software with density function. The software automatically defined the contour of the optic disc as an inside elliptical annulus. The flow density of the elliptical annulus area extending from the inside annulus to $0.75 \mathrm{~mm}$ outward was then separately calculated in six regions (nasal, inferior-nasal, inferior-tempo, superiortempo, superior-nasal and tempo) based on the Garway-Heath Map (figure 1B) on the radial peripapillary capillary (RPC) layer. The software also provided the whole en face flow density, peripapillary and inside disc flow density.

\section{Statistical analysis}

Statistical analysis was performed using an SPSS software package (SPSS for Mac, V.22, IBM/SPSS, Chicago, Illinois, USA). The mean $\pm \mathrm{SD}$ of all the main parameters 
Figure 1 (A) Macular flow density was separately calculated in five regions (fovea, tempo, superior, nasal, and inferior) based on the ETDRS contour. The whole en face flow density was also measured. (B) RPC flow density was separately calculated in six regions (nasal, inferiornasal, inferior-tempo, superior-tempo, superior nasal and tempo) based on the Garway-Heath Map. The whole en face and inside disc flow density was also measured. ETDRS, Early Treatment Diabetic Retinopathy Study; RPC, radial peripapillary capillary.

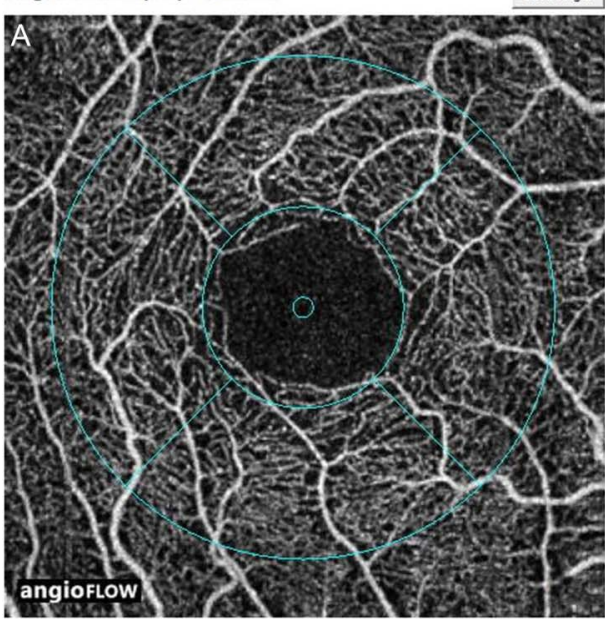

were analysed. One-way analysis of variance (ANOVA) was used to compare the differences of the macular, choriocapillaris and RPC flow densities among the three groups, and in post hoc procedures the Dunnett T3 test was used for multiple comparisons between groups: PM with EM group, PM with HM group, and EM with HM group. The $\chi^{2}$ test was applied to analyse the frequency data of gender. Multiple linear regression analysis was performed to analyse the effect of other independent variables on retinal, choriocapillary density of macular and RPC flow densities, respectively. A value of $\mathrm{p}<0.05$ was considered statistically significant.

\section{RESULTS}

\section{Demographics}

A total of 45 eyes (15 men and 30 women) were included in the EM group. The mean age was 38.3 \pm 13.1 years, mean IOP was $14.8 \pm 2.6 \mathrm{~mm} \mathrm{Hg}$, mean systolic blood pressure (SBP) was $114 \pm 13 \mathrm{~mm} \mathrm{Hg}$, and mean diastolic blood pressure (DBP) was $75 \pm 8 \mathrm{~mm} \mathrm{Hg}$. A total of 41 eyes (11 men and 30 women) were included in the HM group. The mean age was 33.3 \pm 15.0 years, mean IOP was $15.3 \pm 2.6 \mathrm{~mm} \mathrm{Hg}$, mean SBP was $117 \pm 13 \mathrm{~mm} \mathrm{Hg}$ and mean DBP was $78 \pm 9 \mathrm{~mm} \mathrm{Hg}$. A total of 45 eyes (16 men and 29 women) were included in the PM group. The mean age was $38.0 \pm 11.7$ years, mean IOP was $15.3 \pm 2.8 \mathrm{~mm} \mathrm{Hg}$, mean SBP was 114 $\pm 10 \mathrm{~mm} \mathrm{Hg}$ and mean DBP was $77 \pm 7 \mathrm{~mm} \mathrm{Hg}$. No significant differences were found in gender, age, IOP, DBP and SBP among the three groups (table 1).

However, there were significant differences in BCVA, MSE and AL among the three groups (table 1), and multiple comparisons revealed significant differences between the groups. The mean BCVA was 1.0 \pm 0.2 (range 0.8 to 1.5 ), $0.9 \pm 0.2$ (range 0.3 to 1.5 ) and 0.6 \pm 0.3 (range 0.15 to 1.0 ) in the EM, HM and PM groups, respectively $(\mathrm{p}<0.001)$; the mean MSE was 0.07 $\pm 0.35 \mathrm{D}$ (range -0.50 to $0.50 \mathrm{D}),-6.90 \pm 1.23 \mathrm{D}$ (range -6.00 to $-11.00 \mathrm{D}$ ), and $-15.22 \pm 3.79 \mathrm{D}$ (range -8.25 to $-23.00 \mathrm{D})$ in the EM, HM and PM groups, respectively $(\mathrm{p}<0.001)$; and the mean $\mathrm{AL}$ was $23.19 \pm 0.58 \mathrm{~mm}$ (range 22.23 to $24.32 \mathrm{~mm}$ ), $25.93 \pm 0.58 \mathrm{~mm}$ (range 24.76 to $28.19 \mathrm{~mm}$ ), and $29.55 \pm 1.73 \mathrm{~mm}$ (range 27.22 to $33.77 \mathrm{~mm}$ ) in the EM, HM and PM groups, respectively $(\mathrm{p}<0.001$; table 1$)$.

Table 1 Demographic and ocular characteristics of the three groups

\begin{tabular}{|c|c|c|c|c|}
\hline Characteristics & EM (N=45) & HM (N=41) & PM (N=45) & p Value* \\
\hline Gender (male:female) & $15: 30$ & $11: 31$ & $16: 29$ & $0.670 \dagger$ \\
\hline Age (years) & $38.3 \pm 13.1$ & $33.3 \pm 15.0$ & $38.0 \pm 11.7$ & 0.161 \\
\hline BCVA & $1.0 \pm 0.2$ & $0.9 \pm 0.2$ & $0.6 \pm 0.3$ & $<0.001$ \\
\hline IOP (mm Hg) & $14.8 \pm 2.6$ & $15.3 \pm 2.6$ & $15.3 \pm 2.8$ & 0.624 \\
\hline MSE (dioptres) & $0.07 \pm 0.35$ & $-6.90 \pm 1.23$ & $-15.22 \pm 3.79$ & $<0.001$ \\
\hline $\mathrm{AL}(\mathrm{mm})$ & $23.19 \pm 0.58$ & $25.93 \pm 0.58$ & $29.55 \pm 1.73$ & $<0.001$ \\
\hline $\mathrm{SBP}(\mathrm{mm} \mathrm{Hg})$ & $114 \pm 13$ & $117 \pm 13$ & $114 \pm 10$ & 0.504 \\
\hline $\mathrm{DBP}(\mathrm{mm} \mathrm{Hg})$ & $75 \pm 8$ & $78 \pm 9$ & $77 \pm 7$ & 0.221 \\
\hline
\end{tabular}


Table 2 Macular flow density of the three groups

\begin{tabular}{|c|c|c|c|c|}
\hline Variables & $E M(N=45)$ & $\mathrm{HM}(\mathrm{N}=41)$ & $P M(N=45)$ & p Value* \\
\hline Superficial whole en face flow density (\%) & $53.93 \pm 1.72$ & $51.93 \pm 3.38$ & $48.00 \pm 4.10$ & $<0.001$ \\
\hline Superficial fovea flow density (\%) & $29.07 \pm 4.14$ & $28.94 \pm 6.15$ & $30.59 \pm 4.55$ & 0.228 \\
\hline Superficial parafovea flow density (\%) & $55.68 \pm 1.89$ & $54.93 \pm 3.43$ & $50.20 \pm 4.44$ & $<0.001$ \\
\hline Superficial temporal flow density (\%) & $54.46 \pm 2.14$ & $54.33 \pm 3.38$ & $50.63 \pm 4.35$ & $<0.001$ \\
\hline Superficial superior flow density (\%) & $56.69 \pm 2.21$ & $55.96 \pm 3.83$ & $50.66 \pm 5.69$ & $<0.001$ \\
\hline Superficial nasal flow density (\%) & $54.82 \pm 1.91$ & $54.29 \pm 3.28$ & $49.45 \pm 4.70$ & $<0.001$ \\
\hline Superficial inferior flow density (\%) & $56.74 \pm 2.43$ & $55.18 \pm 4.73$ & $50.19 \pm 5.23$ & $<0.001$ \\
\hline Deep whole en face flow density (\%) & $58.25 \pm 1.58$ & $57.76 \pm 2.74$ & $54.59 \pm 4.18$ & $<0.001$ \\
\hline Deep fovea flow density (\%) & $25.64 \pm 4.86$ & $27.01 \pm 7.57$ & $28.86 \pm 6.33$ & 0.056 \\
\hline Deep parafovea flow density (\%) & $61.13 \pm 1.95$ & $62.21 \pm 2.77$ & $58.95 \pm 4.66$ & $<0.001$ \\
\hline Deep temporal flow density (\%) & $59.55 \pm 2.50$ & $61.05 \pm 2.96$ & $58.55 \pm 5.13$ & 0.009 \\
\hline Deep superior flow density (\%) & $62.32 \pm 2.26$ & $63.07 \pm 2.85$ & $58.45 \pm 5.91$ & $<0.001$ \\
\hline Deep nasal flow density (\%) & $60.24 \pm 2.23$ & $61.49 \pm 3.65$ & $59.29 \pm 4.79$ & 0.025 \\
\hline Deep inferior flow density (\%) & $62.40 \pm 2.35$ & $63.33 \pm 3.49$ & $59.50 \pm 6.34$ & $<0.001$ \\
\hline Choriocapillary flow density (\%) & $62.34 \pm 1.05$ & $61.60 \pm 1.65$ & $61.16 \pm 2.61$ & 0.013 \\
\hline
\end{tabular}

Significant $p$ values are in bold.

${ }^{*}$ All calculated by one-way ANOVA.

ANOVA, analysis of variance; EM, emmetropia; HM, high myopia; PM, pathological myopia.

\section{Macular flow density}

Both in the superficial and deep retinal layers significant differences among the three groups were found in all regions, except in the fovea. In the choriocapillaris layer, significant differences were found among the three groups (table 2). The PM group had decreased macular flow density: the superficial whole en face flow densities were $53.93 \pm 1.72 \%, 51.93 \pm 3.38 \%$ and $48.00 \pm 4.10 \%$ in the EM, HM and PM groups, respectively $(\mathrm{p}<0.001)$; the deep whole en face flow densities were $58.25 \pm 1.58 \%$, $57.76 \pm 2.74 \%$ and $54.59 \pm 4.18 \%$ in the EM, HM and PM groups, respectively $(\mathrm{p}<0.001)$; and the choriocapillaris flow densities were $62.34 \pm 1.05 \%, 61.60 \pm 1.65 \%$ and 61.16 $\pm 2.61 \%$ in the EM, HM and PM groups, respectively $(\mathrm{p}=0.013$; table 2$)$.
In the superficial retinal layer, multiple comparisons revealed significant differences between the PM and EM groups, and also between the PM and HM groups, in all regions, while between the $\mathrm{HM}$ and EM groups there was a significant difference in whole en face flow density, but not in the parafovea, temporal, superior, nasal and inferior region (table 3 ).

In the deep retinal layer, between the PM and EM groups, multiple comparisons revealed significant differences in the whole en face, parafovea, superior and inferior region, but not in the temporal and nasal region; between the PM and HM groups, significant differences were found in all regions; between the HM and EM groups, no significant differences were found in all regions (table 3 ).

Table 3 Multiple comparisons of macular flow density between the three groups

\begin{tabular}{|c|c|c|c|}
\hline \multirow[b]{2}{*}{ Variables } & \multicolumn{3}{|l|}{ p Value* } \\
\hline & EM vs HM & EM vs PM & HM vs PM \\
\hline Superficial whole en face flow density & 0.004 & $<0.001$ & $<0.001$ \\
\hline Superficial parafovea flow density & 0.527 & $<0.001$ & $<0.001$ \\
\hline Superficial temporal flow density & 0.996 & $<0.001$ & $<0.001$ \\
\hline Superficial superior flow density & 0.640 & $<0.001$ & $<0.001$ \\
\hline Superficial nasal flow density & 0.750 & $<0.001$ & $<0.001$ \\
\hline Superficial inferior flow density & 0.172 & $<0.001$ & $<0.001$ \\
\hline Deep whole en face flow density & 0.677 & $<0.001$ & $<0.001$ \\
\hline Deep parafovea flow density & 0.115 & 0.016 & $<0.001$ \\
\hline Deep temporal flow density & 0.194 & 0.618 & 0.007 \\
\hline Deep superior flow density & 0.461 & $<0.001$ & $<0.001$ \\
\hline Deep nasal flow density & 0.366 & 0.675 & 0.021 \\
\hline Deep inferior flow density & 0.397 & 0.017 & 0.002 \\
\hline Choriocapillary flow density & 0.047 & 0.020 & 0.731 \\
\hline
\end{tabular}


Table 4 RPC flow density of three groups

\begin{tabular}{|c|c|c|c|c|}
\hline Variables & $E M(N=45)$ & $\mathrm{HM}(\mathrm{N}=41)$ & $P M(N=45)$ & p Value* \\
\hline Optic disc whole en face flow density (\%) & $57.96 \pm 1.95$ & $55.38 \pm 2.69$ & $48.16 \pm 4.16$ & $<0.001$ \\
\hline Optic disc inside disc flow density & $43.87 \pm 7.28$ & $45.15 \pm 9.94$ & $45.38 \pm 8.98$ & 0.699 \\
\hline Optic disc peripapillary flow density (\%) & $65.42 \pm 2.30$ & $62.71 \pm 3.64$ & $52.70 \pm 6.00$ & $<0.001$ \\
\hline Optic disc nasal flow density (\%) & $62.54 \pm 2.85$ & $58.91 \pm 4.02$ & $48.93 \pm 7.84$ & $<0.001$ \\
\hline Optic disc inferior nasal flow density (\%) & $65.68 \pm 3.92$ & $62.02 \pm 6.63$ & $52.22 \pm 8.71$ & $<0.001$ \\
\hline Optic disc inferior tempo flow density (\%) & $68.41 \pm 3.05$ & $66.67 \pm 7.49$ & $57.45 \pm 9.45$ & $<0.001$ \\
\hline Optic disc superior tempo flow density (\%) & $67.52 \pm 3.76$ & $67.30 \pm 4.21$ & $53.91 \pm 9.74$ & $<0.001$ \\
\hline Optic disc superior nasal flow density (\%) & $63.72 \pm 3.57$ & $62.40 \pm 4.12$ & $49.71 \pm 10.46$ & $<0.001$ \\
\hline Optic disc tempo flow density (\%) & $67.24 \pm 3.02$ & $63.79 \pm 8.27$ & $56.73 \pm 9.56$ & $<0.001$ \\
\hline
\end{tabular}

Significant $p$ values are in bold.

*All calculated by the one-way ANOVA.

ANOVA, analysis of variance; EM, emmetropia; HM, high myopia; PM, pathological myopia; RPC, radial peripapillary capillary.

In the choriocapillaris layer, multiple comparisons revealed significant differences between the PM and EM groups, HM and EM groups, but not between the PM and HM groups (table 3 ).

\section{RPC flow density}

Significant differences among the three groups were found in all RPC regions, except inside the disc (table 4). The PM group had decreased RPC flow density: the optic disc's whole en face flow densities were $57.96 \pm 1.95 \%$, $55.38 \pm 2.69 \%$ and $48.16 \pm 4.16 \%$ in the EM, HM and PM groups, respectively $(\mathrm{p}<0.001$; table 4$)$.

Further multiple comparisons revealed significant differences between the PM and EM groups in all regions, and also between the PM and HM groups in all regions. Between the HM and EM groups, significant differences were found in the whole en face, peripapillary, nasal, inferior nasal and tempo region, but not in the inferior tempo, superior tempo and superior nasal region (table 5).

\section{Correlation between AL and BCVA with macular flow density}

By multiple linear regression analysis, both in the superficial and deep retinal layers AL was revealed to be significantly negatively correlated with whole en face flow density (superficial: $\beta=-0.542, p<0.001$; deep: $\beta=-0.282$, $\mathrm{p}=0.002$ ). No significant correlation was found between
$\mathrm{AL}$ and choriocapillary flow density. Both in the superficial and deep retinal layers BCVA was shown to be positively correlated with whole en face flow density (superficial: $\beta=0.194, p=0.021$; deep: $\beta=0.373, p<0.001$ ). BCVA was also shown to be positively correlated with choriocapillary flow density $(\beta=0.291, \mathrm{p}=0.001)$.

\section{Correlation between AL and BCVA with the RPC flow density}

By multiple linear regression analysis, AL was revealed to be negatively correlated with RPC whole en face flow density $(\beta=-0.522, p<0.001)$, while no correlation was found between BCVA and RPC whole en face flow density.

\section{DISCUSSION}

In this study, macular and peripapillary flow densities in pathologically myopic eyes were quantitatively measured by OCT angiography with SSADA.

Various techniques have been used to study ocular blood flow in HM and PM. Avetisov et al reported fluorescein transit time was delayed in pathologically myopic eyes. Akyol et at reported reduced choroidal and retinal blood flow in PM by using CDI. Shimada et $a l^{10}$ found decreased retinal blood flow and vessel diameter in HM by using laser Doppler velocimetry. Benavente-Pérez

Table 5 Multiple comparisons of RPC flow density between the three groups

\begin{tabular}{llll}
\hline & p Value* & & \\
Variables & EM vs HM & EM vs PM & $<0.001$ \\
\hline Optic disc whole en face flow density & $<0.001$ & $<0.001$ & $<01$ \\
Optic disc peripapillary flow density & $<0.001$ & $<0.001$ & $<0.001$ \\
Optic disc nasal flow density & $<0.001$ & $<0.001$ & $<0.001$ \\
Optic disc inferior nasal flow density & $\mathbf{0 . 0 0 9}$ & $<0.001$ & $<0.001$ \\
Optic disc inferior tempo flow density & 0.426 & $<0.001$ & $<0.001$ \\
Optic disc superior tempo flow density & 0.992 & $<0.001$ & $<0.001$ \\
Optic disc superior nasal flow density & 0.312 & & $\mathbf{0 . 0 0 1}$ \\
Optic disc tempo flow density & $\mathbf{0 . 0 4 4}$ & & $\mathbf{0 . 0 0 4}$ \\
\hline Significant $p$ values are in bold. & &
\end{tabular}


et $a l^{11}$ reported reduced retinal artery blood flow in high myopic eyes by using CDI. Spina et $a l^{17}$ reported reduced diameters of the main retinal vessels by using a dynamic vessel analyser in HM and PM. These studies revealed reduced choroidal and retinal blood flow in HM and PM. But these techniques were limited to measuring ocular blood flow in large vessels. OCT angiography was proven to be able to document in vivo choroidal and retinal vasculature including microvasculature in a non-invasive and quantitative way, and enabled high-resolution assessment of early and subtle changes in macular and papillary vasculature. Good repeatability and reproducibility of SSADA OCT angiography were reported in previous studies. ${ }^{15} 161819$ There are few quantitative studies of vascular density by OCT angiography in PM. Wang et $a t^{20}$ studied macular and peripapillary perfusion by OCT angiography only in high myopic eyes, while this study focused on pathologically myopic eyes.

In agreement with previous studies, in this study the PM and HM groups had decreased choriocapillary flow densities compared with the EM group; however, no significant difference was found between the PM and HM groups. One study showed that in pathologically myopic eyes severe choriocapillaris atrophy was present and the normal anatomic levels were displaced, ${ }^{7}$ but the choriocapillaris layer was still autosegmented by software from RPE with an RPE offset of 31-59 $\mu \mathrm{m}$. Thus the flow density actually measured might include the mediumsize choroidal vessels or even the deeper large-size choroidal vessels instead of choriocapillaris only; that might explain why there is no significant difference in choriocapillary flow density between the PM and HM groups in this study.

Both in the superficial and deep retina, decreased macular flow density was found in the PM group compared with the HM and EM groups, except in the foveal region and the deep temporal and deep nasal region. Between the HM and EM groups, except for superficial whole en face flow density, no differences were found both in the superficial and deep retina, in accordance with a previous study. ${ }^{20}$ These results indicate that decreased macular flow density was only found in pathologically myopic eyes, but not in HM eyes. Maybe the foveal region was mainly an avascular zone, so it is less sensitive to detecting some differences among groups. It is hard to explain why no differences were found in the deep temporal and deep nasal regions; perhaps a larger sample was needed.

Radial peripapillary capillaries (RPCs) comprise a unique network of capillary beds within a retinal nerve fibre layer (RNFL) and play a critical role in providing nutritional requirements of retinal ganglion cell axons. Previous studies have reported that RPC capillary density was significantly greater in the arcuate fibre region (inferior temporal and superior temporal regions). The unique metabolic demands of neuronal subcompartments may influence the morphometric features of regional capillary networks, and differences in capillary density may have important correlations with neuronal function in the human retina. ${ }^{21}$ Wang et $a l^{20}$ reported decreased peripapillary perfusion in high myopic eyes by OCT angiography. In this study, RPC flow density was significantly decreased in the PM group compared with the HM and EM groups, except the inside disc region. Perhaps the complex composition of large vessels inside the disc made it less sensitive to detecting the differences among groups. Our results also revealed RPC whole en face flow density was significantly decreased in the HM group compared with the EM group in the peripapillary, nasal, inferior nasal and tempo regions, but not in the inferior temporal, superior temporal and superior nasal regions. This result indicated that the RPC flow density in high myopic eyes might be preserved in the arcuate fibre region to satisfy the metabolic demands of the RNFL. As previously mentioned, no significant decreases were found in macular flow densities of high myopic eyes; such relative preservation of retinal vascular flow density might ensure the normal visual function of high myopic eyes.

Spina et $\mathrm{al}^{17}$ reported reduced dimensions of the main retinal vessels appeared inversely related to $\mathrm{AL}$, and Yang et $a l^{22}$ reported ocular blood flow was negatively related to AL. In accordance with these studies, this study found that both macular and RPC flow densities were negatively related to AL by multiple linear regression analysis. The mechanism of decreased retinal and choroidal perfusion in pathological myopic eyes is still unclear. Studies demonstrated that retinal and choroidal thickness decreased in HM and PM. ${ }^{23-25}$ Man et $a l^{26}$ highlighted how longer eyes had decreased retinal function and oxygen consumption. It was commonly thought that in pathologically myopic eyes excessive elongation of the eyeball could cause thinning of the retina and choroid, and the thinning of these tissues might decrease the oxygen demand and consequently decrease the blood circulation. But whether decreased blood flow was only a consequence of chorioretinal atrophy or was involved in the development of PM is still unknown. Moreover, our results demonstrated that only macular flow density was positively related to BCVA. The results confirmed that the macular vasculature system was essential to visual function, suggesting that improvement of macular perfusion might increase the visual function of PM. However, the mechanisms of decreased ocular perfusion and how to preserve or improve perfusion of PM requires further study.

Image artefacts were not uncommon in OCT angiography, and must be taken into account for appropriate interpretation. ${ }^{27}$ Although OCT angiography has proven to be an effective and non-invasive method for monitoring macular and peripapillary perfusion, in this study we noticed that the use of OCT angiography was limited in pathologically myopic eyes. First, we know that ocular movements, even subtle, can cause significant artefacts on OCT angiography; thus some pathologically myopic eyes with 
poor visual acuity and fixation could hardly obtain a good quality image. Second, when severe chorioretinal lesions, such as chorioretinal atrophy, foveoschisis or retinal detachment, were present and the normal anatomic levels were displaced, an error of automated segmentation by OCT angiography or projection artefacts might occur. Third, the OCT signal might be compromised by severe vitreous opacities, which are common in PM.

This study had some limitations: it is a single-centre cross-sectional study with a monoracial background, involving a limited number of cases; a further study with a larger sample size might tell us more about the ocular vasculature in PM. The links between vascular changes with visual functionality in pathologically myopic eyes are still unclear; thus, a follow-up study on the relationship between ocular circulation using OCT angiography with retinal function might be able to improve our understanding of the pathophysiology in PM.

In conclusion, this study aimed to investigate the vascular flow density in PM by using OCT angiography; the results demonstrated a reduced macular and peripapillary flow density in pathologically myopic eyes. No significant decrease of retinal flow density in the macular and arcuate fibre region was found in high myopic eyes compared with emmetropic eyes. In addition, the macular and peripapillary flow density was shown to be negatively related to AL and macular flow density was positively related to BCVA. It is worth noting that there are some limitations in the use of OCT angiography in PM. Improvement of macular perfusion might increase the visual function of PM, but mechanisms of the decreased ocular perfusion and how to preserve or improve perfusion in pathologically myopic eyes requires further study.

Contributors WW and JM conceived of and designed the study. JM, AD and XW performed the study. JM and SC analysed the data. WW and JM wrote the manuscript.

Funding This work was supported by the Beijing Municipal Administration of Hospitals' Ascent Plan(code: DFL20150201); Science \& Technology Project of Beijing Municipal Science \& Technology Commission (code: Z151100001615052); National Natural Science Foundation of China (Nr.81570891); Beijing Municipal Administration of Hospitals Clinical Medicine Development of Special Funding Support (code: ZYLX201307); National Natural Science Foundation of China (Nr. 81272981); Beijing Natural Science Foundation (Nr. 7151003); Advanced Health Care Professionals Development Project of Beijing Municipal Health Bureau No. 2014-2-003.

Competing interests None declared.

Patient consent Obtained.

Ethics approval This study was approved by the Medical Ethics Committee of the Beijing Tongren Hospital.

Provenance and peer review Not commissioned; externally peer reviewed.

Data sharing statement No additional data are available.

Open Access This is an Open Access article distributed in accordance with the Creative Commons Attribution Non Commercial (CC BY-NC 4.0) license, which permits others to distribute, remix, adapt, build upon this work noncommercially, and license their derivative works on different terms, provided the original work is properly cited and the use is non-commercial. See: http:// creativecommons.org/licenses/by-nc/4.0/

\section{REFERENCES}

1. Cedrone C, Nucci C, Scuderi G, et al. Prevalence of blindness and low vision in an Italian population: a comparison with other European studies. Eye (Lond) 2006;20:661-7.

2. Buch $\mathrm{H}$, Vinding T, Nielsen NV. Prevalence and causes of visual impairment according to World Health Organization and United States criteria in an aged, urban Scandinavian population: the Copenhagen City Eye Study. Ophthalmology 2001;108: 2347-57.

3. Xu L, Wang Y, Li Y et al. Causes of blindness and visual impairment in urban and rural areas in Beijing: the Beijing Eye Study. Ophthalmology 2006;113:1134.e1-11. e1111.

4. Hsu WM, Cheng CY, Liu JH, et al. Prevalence and causes of visual impairment in an elderly Chinese population in Taiwan: the Shihpai Eye Study. Ophthalmology 2004;111:62-9.

5. Iwase A, Araie M, Tomidokoro A, et al. Prevalence and causes of low vision and blindness in a Japanese adult population: the Tajim Study. Ophthalmology 2006;113:1354-62. e1351.

6. Ohno-Matsui K, Kawasaki R, Jonas JB, et al. International photographic classification and grading system for myopic maculopathy. Am J Ophthalmol 2015;159:877-883.e7.

7. Jonas JB, Xu L. Histological changes of high axial myopia. Eye (Lond) 2014;28:113-7.

8. Avetisov ES, Savitzkaya NF. Some features in ocular microcirculation in myopia. Ann Ophthalmol 1977;9:1261-4.

9. Akyol N, Kükner AS, Ozdemir T, et al. Choroidal and retinal blood flow changes in degenerative myopia. Can J Ophthalmol 1996;31:113-19.

10. Shimada N, Ohno-Matsui K, Harino S, et al. Reduction of retinal blood flow in high myopia. Graefes Arch Clin Exp Ophthalmol 2004:242:284-8.

11. Benavente-Pérez A, Hosking SL, Logan NS, et al. Ocular blood flow measurements in healthy human myopic eyes. Graefes Arch Clin Exp Ophthalmol 2010;248:1587-94.

12. Spaide RF, Klancnik JM, Cooney MJ. Retinal vascular layers imaged by fluorescein angiography and optical coherence tomography angiography. JAMA Ophthalmol 2015;133:45-50.

13. Quaranta M, Arnold J, Coscas G, et al. Indocyanine green angiographic features of pathologic myopia. Am J Ophthalmol 1996;122:663-71.

14. Jia Y, Tan O, Tokayer J, et al. Split-spectrum amplitude-decorrelation angiography with optical coherence tomography. Biomed Opt Express 2012;20:4710-25.

15. Jia $\mathrm{Y}$, Wei E, Wang X, et al. Optical coherence tomography angiography of optic disc perfusion in glaucoma. Ophthalmology 2014;121:1322-32.

16. $\mathrm{Yu}$ J, Jiang $\mathrm{C}$, Wang $\mathrm{X}$, et al. Macular perfusion in healthy Chinese: an optical coherence tomography angiogram study. Invest Ophthalmol Vis Sci 2015;56:3212-17.

17. Spina CL, Corvi F, Bandello F, et al. Static characteristics and dynamic functionality of retinal vessels in longer eyes with or without pathologic myopia. Graefes Arch Clin Exp Ophthalmol 2016;254:827-34.

18. Wang $\mathrm{X}$, Jiang $\mathrm{C}, \mathrm{Ko} \mathrm{T}$, et al. Correlation between optic disc perfusion and glaucomatous severity in patients with open-angle glaucoma: an optical coherence tomography angiography study. Graefes Arch Clin Exp Ophthalmol 2015;253:1557-64.

19. Wei E, Jia $Y$, Tan $O$, et al. Parafoveal retinal vascular response to pattern visual stimulation assessed with OCT angiography. PLOS One 2013;8:e81343.

20. Wang $X$, Kong $X$, Jiang $C$, et al. Is the peripapillary retinal perfusion related to myopia in healthy eyes? A prospective comparative study. BMJ Open 2016;6:e010791.

21. Tan PE, Yu PK, Balaratnasingam C, et al. Quantitative confocal imaging of the retinal microvasculature in the human retina. Invest Ophthalmol Vis Sci 2012;53:5728-36.

22. Yang YS, Koh JW. Choroidal blood flow change in eyes with high myopia. Korean J Ophthalmol 2015;29:309-14.

23. Ohno-Matsui K, Lai TY, Lai CC, et al. Updates of pathologic myopia. Prog Retin Eye Res 2016;52:156-87.

24. Wang S, Wang Y, Gao X, et al. Choroidal thickness and high myopia: a cross-sectional study and meta-analysis. BMC Opthalmol 2015;15:70.

25. Cicinelli MV, Pierro L, Gagliardi M, et al. 2015. Optical coherence tomography and pathological myopia: an update of the literature. Int Ophthalmol 2015;35:897-902.

26. Man RE, Lamoureux EL, Taouk Y, et al. Axial length, retinal function, and oxygen consumption: a potential mechanism for a lower risk of diabetic retinopathy in longer eyes. Invest Ophthalmol Vis Sci 2013;54:7691-8.

27. Spaide RF, Fujimoto JG, Waheed NK. Image artifacts in optical coherence tomography angiography. Retina 2015;35:2163-80. 